\title{
Optimization of the anode shape for the electroplating coating on long thin-walled detail taking into account the ohmic potential drop
}

\author{
Inna Solovjeva ${ }^{1 *}$, Denis Solovjev ${ }^{2}$, Viktoriya Konkina ${ }^{1}$, and Yuri Litovka ${ }^{1}$ \\ ${ }^{1}$ Tambov State Technical University, 392000, Sovetskaya, 106, Tambov, Russia \\ ${ }^{2}$ Tambov State University named after G.R. Derzhavin, 392036, Internatsionalnaya, 33, Tambov, \\ Russia
}

\begin{abstract}
The article discusses the problem of optimizing the anode shape to reduce the non-uniformity of the electroplating coating for a long thinwalled detail. An increase in the non-uniformity of the coating due to the ohmic potential drop in the electrodes body is characteristic of such details. The problem of optimizing the anode shape is formulated to minimize the non-uniformity of the electroplating coating. The mathematical model of the electroplating process has been developed, which takes into account the ohmic potential drop in the electrodes body. The problem of optimizing the anode shape is solved by the example of zinc electroplating process in an alkaline electrolyte, taking into account the ohmic potential drop in the electrodes body and without it.
\end{abstract}

\section{Introduction}

Electroplating coatings are used to modify detail surface properties and protect them from corrosion [1]. Uniformity is one of the key factors in determining the quality of an electroplating coating [2]. Non-uniformity leads to the detail rejection (if the coating thickness is less than the specified value) and an increase in the cost of the electroplating process (if the coating thickness is greater than the specified value). The non-uniformity of the electroplating coating is caused by different strengths of the electric field on the detail surface immersed in an electrolyte solution. Shaped anodes are one of the ways to reduce the coating non-uniformity. The article [3] is devoted to the research the effect of the number, size and anodes location in an electroplating bath on the non-uniformity of the resulting coating. In the article [4] the anode shape is computed over a number of predefined time steps by convection of its surface with a velocity proportional and in the direction of the local electrode shape change rate. The article [5] is devoted to the research of the anode shape dependence on the alignment of the distance between the opposing local anode and cathode (detail) sections. However, the inhomogeneity of the electric field is enhanced by the ohmic potential drop in the electrodes body when electroplating long thinwalled details, increasing the non-uniformity of the coating [6]. From the analysis it follows that there are no researches of the anode shape dependence for the process of coating long

*Corresponding author: good.win32@yandex.ru 
thin-walled details using mathematical models that take into account the ohmic potential drop in the electrodes body.

The search for the optimal anode shape for the electroplating coating on long thinwalled detail, taking into account the ohmic potential drop in the electrodes body is the article purpose.

\section{Materials and methods}

Let us formulate the optimization problem for finding the anode shape. Let in the electroplating bath space, determined by the Cartesian coordinates $(x, y, z)$, the anode is represented in the form of $N$ nodal points $A_{1}\left(x_{1}, y_{1}\right), A_{2}\left(x_{2}, y_{2}\right), \ldots, A_{N}\left(x_{N}, y_{N}\right)$. Evaluation of non-uniformity coating is carried out by the Kadaner's criterion:

$$
R=\frac{1}{S_{C}} \int_{S_{C}} \frac{\delta(x, y, z)-\delta^{\min }}{\delta^{\min }} d S_{C},
$$

where $S_{C}$ is the cathode surface (long thin-walled detail); $\delta$ is the coating thickness; min is the minimum value.

It is necessary to find the coordinate values of the nodal points $A_{i}\left(x_{i}, y_{i}\right)(i=1, \ldots, N)$, at which the non-uniformity criterion (1) will be minimal under the restriction on the minimum value of the coating thickness on the cathode surface:

and anode surface dimensions:

$$
\delta^{\min }=\delta^{\text {spec }},
$$

$$
\begin{aligned}
& 0 \leq x_{i} \leq L_{x}, \\
& 0 \leq y_{i} \leq L_{y},
\end{aligned}
$$

where spec is the specified value; $L_{x}, L_{y}$ is the length and width of the electroplating bath.

Let's compose a mathematical model for connecting the coordinates of nodal points on the anode shape with the non-uniformity criterion (1).

The coating thickness is calculated by the Faraday's law:

$$
\delta(x, y, z)=\frac{k \eta t}{\rho} j_{C}(x, y, z),
$$

where $k$ is the electrochemical equivalent; $\rho$ is the metal density; $\eta$ is the the metal current output; $t$ is the time; $j_{C}$ is the cathode current density.

The current density at the electrodes is calculated by the Ohm's law:

$$
\begin{aligned}
& j_{C}(x, y, z)=-\left.\chi \nabla \varphi(x, y, z)\right|_{S_{C}}, \\
& j_{A}(x, y, z)=\left.\chi \nabla \varphi(x, y, z)\right|_{S_{A}},
\end{aligned}
$$

where $S_{A}$ is the anode surface; $\chi$ is the specific conductance; $\varphi$ is the electric field potential in electrolyte solution.

The electric field potential in electrolyte solution is calculated by the Laplace's equation:

$$
\partial^{2} \varphi(x, y, z) / \partial x^{2}+\partial^{2} \varphi(x, y, z) / \partial y^{2}+\partial^{2} \varphi(x, y, z) /\left.\partial y^{2}\right|_{V_{E L}}=0
$$

with boundary conditions:

$$
\begin{gathered}
\partial \varphi(x, y, z) /\left.\partial \vec{n}\right|_{S_{I N S}}=0, \\
\varphi(x, y, z)+F_{A}\left(\left.j_{A}(x, y, z)\right|_{S_{A}}=\varphi_{A}(x),\right. \\
\varphi(x, y, z)-\left.F_{C}\left(j_{C}(x, y, z)\right)\right|_{S_{C}}=\varphi_{C}(x),
\end{gathered}
$$


where $V_{E L}$ is the electrolyte space; $S_{I N S}$ is the insulator surface; $\vec{n}$ is the normal to surface; $F_{A}, F_{C}$ are the functions of anodic and cathodic polarization; $\varphi_{A}, \varphi_{C}$ are the potential distribution functions on the anode and cathode surfaces.

Taking into account the ohmic potential drop on a long thin-walled detail is a feature of equations (5)-(11), which is reflected in the right-hand side of boundary conditions (10) and (11). Consider an algorithm for determining the potential distribution function on the anode and cathode surfaces depending on their length.

The bath space $S_{I N S}$ with electrolyte $V_{E L}$ and electrodes $S_{A}$ and $S_{C}$ is represented in the form of an equivalent circuit diagram (fig. 1). Total circuit resistance is calculated using serial and parallel conversion and Kirchhoff's laws [7].

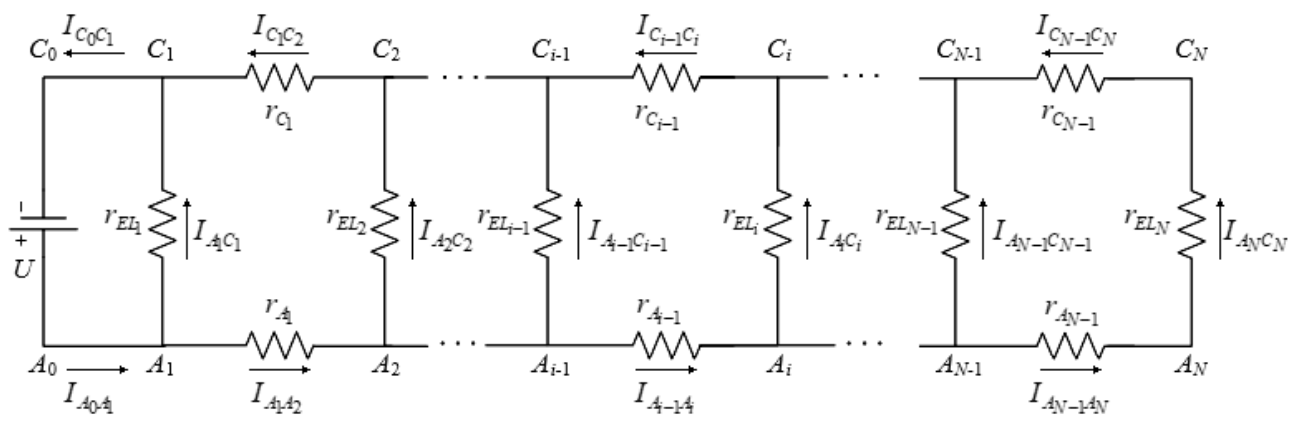

Fig. 1. Equivalent circuit diagram of the electroplating bath space with electrodes and electrolyte

The $i$-th section resistance of the electrolyte is determined as:

$$
r_{E L_{i}}=\frac{l_{i}}{\chi d x d z},
$$

where $l_{i}$ is the distance between the $i$-th section of the anode and cathode; $d x, d z$ are the steps on $x$ and $z$ coordinates.

The $i$-th section resistances of the anode and cathode are determined as:

$$
\begin{aligned}
& r_{A_{i}}=\rho_{A} \frac{d x}{h_{A_{i}} d z}, \\
& r_{C_{i}}=\rho_{C} \frac{d x}{h_{C_{i}} d z},
\end{aligned}
$$

where $\rho_{A}, \rho_{C}$ are the specific resistances of the anode and cathode; $h_{A_{i}}, h_{C_{i}}$ are the wall thicknesses of the anode and cathode.

In general, the iterative process of calculating the discrete values of the electrode potentials included in the boundary conditions (10) and (11) is described as:

$$
\begin{gathered}
\varphi_{A_{i}}=\varphi_{A_{i-1}}-U_{A_{i-1} A_{i}}, \\
\varphi_{C_{i}}=\varphi_{C_{i-1}}+U_{C_{i-1} C_{i}}, \\
U_{A_{i-1} A_{i}}=I_{A_{i-1} A_{i}} r_{A_{i}}, \\
U_{C_{i-1} C_{i}}=I_{C_{i-1} C_{i}} r_{C_{i}}, \\
I_{A_{i-1} A_{i}}=I_{A_{i-2} A_{i-1}}-I_{A_{i-1} C_{i-1}}, \\
I_{C_{i-1} C_{i}}=I_{C_{i-2} C_{i-1}}-I_{A_{i-1} C_{i-1}}, \\
I_{A_{i-1} C_{i-1}}=\frac{U_{A_{i-1} C_{i-1}}}{r_{E L_{i-1}}}=\frac{\varphi_{A_{i-1}}-\varphi_{C_{i-1}}}{r_{E L_{i-1}}},
\end{gathered}
$$

with initial conditions: 


$$
\begin{gathered}
\varphi_{A_{0}}=U, \\
\varphi_{C_{0}}=0, \\
I_{A 0 A_{1}}=I_{C 0 C_{1}}=I,
\end{gathered}
$$

where $U$ is the supply voltage; $I$ is the total current.

Calculation of equations (5)-(24) can take a considerable time depending on the grid spacing in coordinates $(x, y, z)$ and the electroplating bath size. This greatly complicates the application of gradient optimization methods. In addition, the error in calculating the derivative increases significantly due to the approximation of the nodal points coordinates $A_{i}\left(x_{i}, y_{i}\right)(i=1, \ldots, N)$ to the grid nodes. The method of local variations is proposed for finding the criterion (1) minimum [8]. The method doesn't include the calculation of derivatives and at the same time retains the possibility of a sufficiently fast movement to a minimum.

\section{Experimental section}

Let us consider the zinc electroplating process in an alkaline electrolyte of the long thinwalled detail with front surface $S_{C}=15 \cdot 10^{3} \mathrm{~cm}^{2}$ and wall thickness $h_{C_{i}}=$ const $=1 \mathrm{~cm}$. The ohmic potential drop on a long thin-walled part was taken into account at $d x=d z=1 \mathrm{~cm}$. The values list of constants and mode parameters is presented in Table 1.

Table 1. The values list of constants and mode parameters.

\begin{tabular}{|c|c|c|c|c|c|c|c|c|c|}
\hline $\begin{array}{c}\text { Symbol, } \\
\text { unit of } \\
\text { measurement }\end{array}$ & $\begin{array}{c}\rho, \\
\mathrm{kg} / \mathrm{cm}^{3}\end{array}$ & $\begin{array}{c}k, \\
\mathrm{~kg} /(\mathrm{A} \cdot \mathrm{h})\end{array}$ & $\eta$ & $\begin{array}{c}\chi, \\
1 /(\Omega \mathrm{cm})\end{array}$ & $\begin{array}{c}U, \\
\mathrm{~V}\end{array}$ & $\begin{array}{c}S_{I N S}, \\
\mathrm{~cm}^{2}\end{array}$ & $\begin{array}{c}V_{E L}, \\
1\end{array}$ & $\begin{array}{c}\rho_{A}, \\
\Omega \mathrm{cm}^{2} / \\
\mathrm{cm}\end{array}$ & $\begin{array}{c}\rho_{C}, \\
\Omega \mathrm{cm}^{2} / \\
\mathrm{cm}\end{array}$ \\
\hline Value & 7.13 & $1.22 \cdot 10^{-3}$ & 0.98 & 0.435 & 5 & $8 \cdot 10^{4}$ & $2 \cdot 10^{3}$ & $1.5 \cdot 10^{-5}$ & $0.6 \cdot 10^{-5}$ \\
\hline
\end{tabular}

The functions of anodic and cathodic polarization obtained as an approximation result take the form:

$$
\begin{gathered}
F_{A}\left(j_{A}(x, y, z)\right)=0.935 j_{A}(x, y, z), \\
F_{C}\left(j_{C}(x, y, z)\right)=-\left(0.188+0.3 \ln \left(0.43 j_{C}(x, y, z)\right) .\right.
\end{gathered}
$$

The optimization problem (1) was solved at $N=9$ and $N=17$ with anode wall thickness $h_{A_{i}}=$ const $=0.5 \mathrm{~cm}$. The values list of restrictions (2)-(4) is presented in Table 2.

Table 2. The list of restrictions.

\begin{tabular}{|c|c|c|c|}
\hline Symbol, unit of measurement & $\delta^{s p e c}, \mu \mathrm{m}$ & $L_{x}, \mathrm{~cm}$ & $\begin{array}{c}L_{y}, \\
\mathrm{~cm}\end{array}$ \\
\hline Value & 20 & 200 & 100 \\
\hline
\end{tabular}

The distribution of the coating was compared with the coating obtained using a flat anode with a front surface $S_{A}=1440 \mathrm{~cm}^{2}$.

\section{Results and discussion}

The approximation of the discrete values for the potential distribution functions on the anode and cathode surfaces take the form:

$$
\begin{aligned}
& \varphi_{A}(x)=4.891-0.000362 x+0.111 e^{0.00115 x}, \\
& \varphi_{C}(x)=0.155+0.000454 x-0.155 e^{0.00108 x} .
\end{aligned}
$$


The found surfaces of the shaped anodes and it's location in the electroplating bath space have the form shown in fig. 2 .

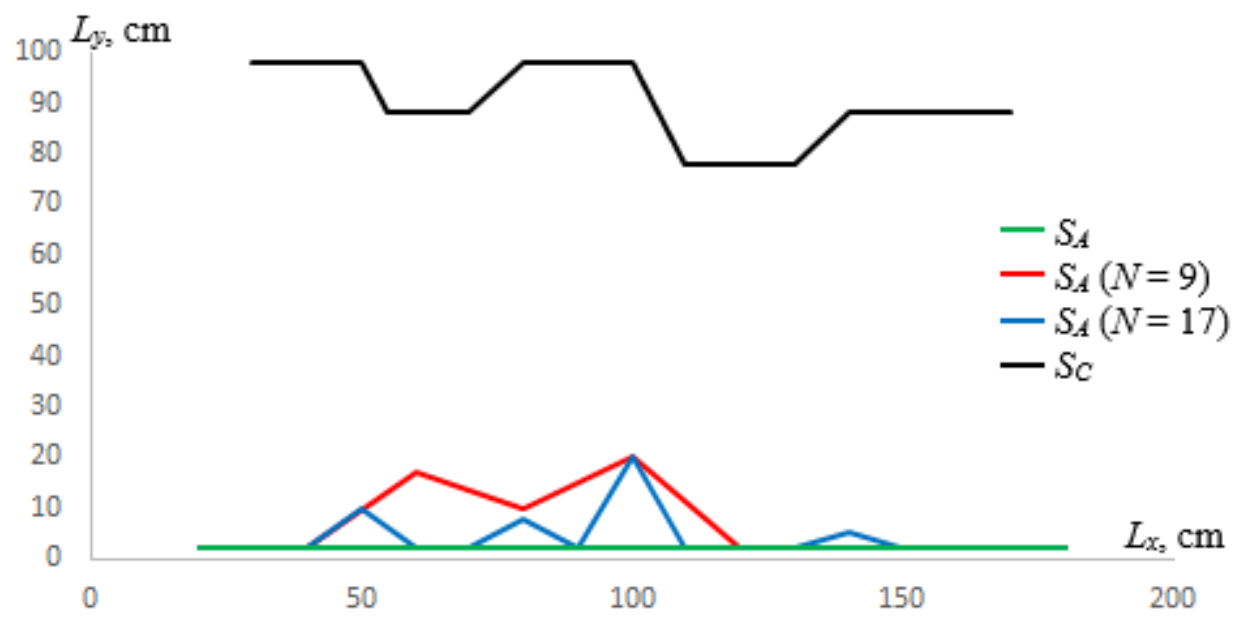

Fig. 2. The found surfaces of the shaped anodes and it's location in the electroplating bath space

The front surfaces of the shaped anodes were $S_{A}=17600 \mathrm{~cm}^{2}$ and SA $=17190 \mathrm{~cm}^{2}$ at $N$ $=9$ and $N=17$. The coating deposition time was $t=47 \mathrm{~min}$ and $t=49 \mathrm{~min}$, which is $21.27 \%$ and $16.13 \%$ less than when using a flat anode. This is due to the increase in the front surface area of shaped anodes compared to a flat anode. The coating thickness distribution by length $L_{C}$ of the cathode front surface $S_{C}$ when using a flat anode and shaped anodes is shown in fig. 3.

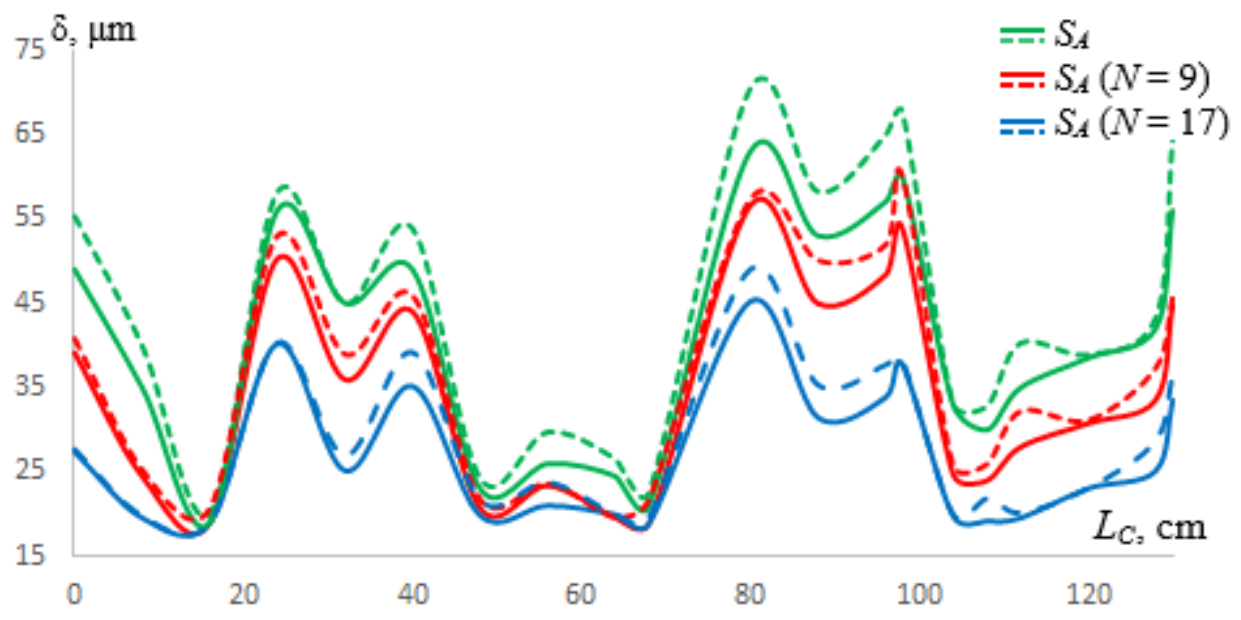

Fig. 3. The coating thickness distribution by length of the cathode front surface with allowance (solid line) and without allowance (dashed line) for ohmic potential drop using a flat anode and shaped anodes

The non-uniformity criterion has the value $R=0.814682$ and $R=0.4173915$ as a result of using a shaped anode at $N=9$ and $N=17$, which is $28.83 \%$ and $63.54 \%$ less than when using a flat anode. This is due to the alignment of the interelectrode distance.

The coating thickness obtained without taking into account the ohmic potential drop by length of the electrodes turned out to be $5.95-8.6 \%$ more than the coating thickness 
obtained taking into account the ohmic potential drop. This is due to the traditional assumption of the electrode potentials constancy when simulating electroplating processes $[9,10]$.

\section{Conclusion}

When coating long thin-walled details using similar anodes, the electrical potential changes by length of the electrodes, since they have high resistance. Thus, the calculated coating thickness will be greater than the actual thickness, if you don't take into account the ohmic potential drop in long thin-walled electrodes. This can lead to the appearance of areas on detail with a coating thickness less than a specified one, that is, a detail defect.

The reported study was funded by RFBR, project number 20-37-90019.

\section{References}

1. Y.D. Gamburg, G. Zangari, Theory and practice of metal electrodeposition (Springer, New York, 2011)

2. Y.-J. Tan, K.Y. Lim, Understanding and improving the uniformity of electrodeposition, Surf Coat Tech, v. 167, pp. 255-262 (2003)

3. F. Druesne, M. Afzali, Electroplating simulation and design tool, Proc Inst Mech Eng B-J Eng Ma, v. 217. pp. $705-707$ (2003)

4. T. Vasile, M. Purcar, A. Avram, C. Munteanu, R. Chereches, L. Grindei, Simulation of the electrode shape change in electrochemical machining based on the level set method, EPJ AP, v. 58. 11301 (2012)

5. V. Volgin, V. Lyubimov, I. Gnidina, T. Kabanova, A. Davydov, Effect of Anode Shape on Uniformity of Electrodeposition onto Resistive Substrates, Electrochimica Acta, v. 230, pp. 382-390 (2017)

6. Y.V. Litovka, S.Y. Denisov, Calculation of the distribution of an electroplated coating over the surface of large-size articles, Russ J Appl Chem, v. 83, pp. 841-845 (2010)

7. C.R. Paul, Fundamentals of Electric Circuit Analysis (John Wiley \& Sons, New York, 2001)

8. R. Fletcher, Practical Methods of Optimization (John Wiley \& Sons, Chichester, 1987)

9. K. I. Popov, P.M. Živković, N.D. Nikolić, A mathematical model of the current density distribution in electrochemical cells, J Serb Chem Soc, v. 76(6), pp. 805-822 (2011)

10. D. Solovjev, I. Solovjeva, V. Konkina, Mathematical modelling and optimization of the electroplating process with a rotating cathode to reduce the non-uniformity of the coating thickness, MATEC Web Conf, v. 298, 00014 (2019) 